\title{
Simulation of Parachute Ripcord Process Based on Fluid-Solid Coupling
}

\author{
Li Naitian*, Tong Zongkai, Gao yong \\ Beijing Electro-Mechanical Engineering Institute, Beijing 10074, P. R. China
}

flyntl@163.com

\begin{abstract}
Keywords: Parachute balloon; fluid-structure coupling; impact properties; explicit dynamics; LS-DYNA

Abstract. In the process of inflation and stabilization descent for the parachute, a coupling effect is generated between the the structure deformation and changes in the flow field around the canopy, which is significant important as well as the dynamic effect of the parachute. Besides, the peak value of the loads on the parachute often occurs in the inflation process where the canopy shape is dramatically changed. Therefore, it is necessary to study the impacting process of the parachute airbag. In this paper, the Arbitrary Lagrangian Eulerian algorithm (ALE) and fluid-structure coupling function of the explicit dynamics program LS-DYNA is used to simulate the stabilizing droplet process of the parachute. The results show that the modeling method and parameter control is feasible. On this basis, the finite element model of the parachute without parasailing is established, and then its inflatable process is simulated, obtaining the impact properties.
\end{abstract}

\section{Introduction}

Parachute - airbag recovery mode has been widely used in aircraft recovery field since this method owns the advantages of easy operation [1]. Currently domestic researches on the airbag cushion characteristic mainly focus on theoretical and experimental methods, and the numerical simulation method recovery results are rare. Because of its advantages of economy, flexibility and repeatability, the numerical simulation method is used more and more widely. In other countries, the numerical simulation calculation method has been successfully adapted in automobile airbags, recovery and other spacecraft.

A dynamics model of the parachute combination is established in references [2, 3], where the parachutes and recovered material are simplified as rigid, and the parachute cord is considered as a rigid rod with concentrated multistage mass. The reference [4] divided the parachute into several plate elements, which is connected by a hinge between the panel units. References $[5,6]$ established a two-particle model for the parachute with certain assumptions. However, most of the related parachute system simulation analysis works reported in those above references are concentrated in a certain stage, which cannot meet the demands of aircraft recycling comprehensive analysis of the entire process.

In the inflation process and stabilization descent of a parachute, the deformation changes of the canopy is mutually coupled with the around flow field. The canopy structural deformation makes shape of canopy become from the elongated body to a bluff body, which causing a significant change in the aerodynamic shape and has an impact on the flow field around the canopy. At the same time, in many cases parachute dynamic effect is very important, especially parachute peak load occur often dramatic changes in canopy shape of the inflation process, which also requires us to structure model the flow field around the model canopy and canopy of coupling. In this paper, the LS-DYNA with the function of fluid-solid coupling is used for the parachute deployment process simulation.

\section{Simulation of parachute inflation process based on fluid-solid coupling}

\subsection{Fluid-solid coupling model and controlling algorithm}

The parachute inflatable model includes parachute canopy, risers and flow field, where the main parachute is a single system with the type of ring parasailing, merged with three straps after connecting, and the three connection points in structure are connected with straps. The characteristics 
of the fluid material is described through the constitutive material model and the equation of state EOS, which offers air material models in the program * MAT_NULL to describe the behavior of a material having a fluid (such as air, water, etc.).

The fluid is modeled with solid elements, which is a single point of integration with central ALE element. This tip can not only reduce the amount of elements but also ensure the parachute moving space. The flow field shape is cylindrical. There are three treatment methods in LS-DYNA to realize the fluid and solid coupling, which are merging the interface node between fluids and solids, contact algorithm and Euler - Lagrange coupling algorithm. In this paper, the Euler - Lagrange coupling algorithm is used to couple the fluid and solid, by using the keyword * CONSTRAINED_LAGRANGE_IN_SOLID in the program implementation. The penalty function is used in order to avoid penetration, and an integration point is four. The * CONTROL_ALE is used for ALE algorithm control, while the fluid delivery algorithm is Van Leer, which is a second-order accurate algorithm. For fluid-structure coupling large deformation, hourglass must be controlled to ensure the proportion of total energy can hourglass maintained at $5 \%$ or less, LS-DYNA provides a variety of methods hourglass control, simulation herein using standard viscous hourglass control according to relevant research experience

\subsection{Parameter validation of Stable decline phase}

Figure 1 (a) is the finite element model of parachute in the stabilize descent phase, and Figure 1 (b) is the initial state flow model.

To simplify the calculation, the shell elements are used in simulation and ensure its quality characteristics consistent with reality. The material of parachute, parachute cord, connecting belts is defined as polyamide-6 fiber, while the gas is defined as empty. The density is $1.0 \mathrm{~kg} \cdot \mathrm{m}^{-3}$, and the dynamic viscosity coefficient is $1.75 \times 10^{-5} \mathrm{~N} \bullet \mathrm{s} \bullet \mathrm{m}^{-1}$. The Flow field height is $100 \mathrm{~m}$, and the diameter is $60 \mathrm{~m}$. Parachute is modeled by shell elements. The role of a parachute and fluid coupling is considered in this paper. This problem is solved by LS-DYNA971 version, and $16 \mathrm{CPU}$ is taken for 13 hours when five seconds process is simulated.
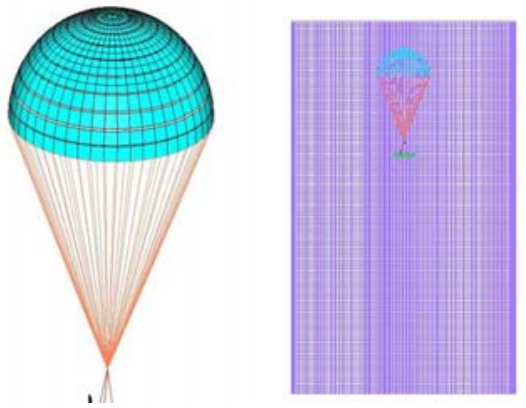

Figure 1 Finite element in the simulation

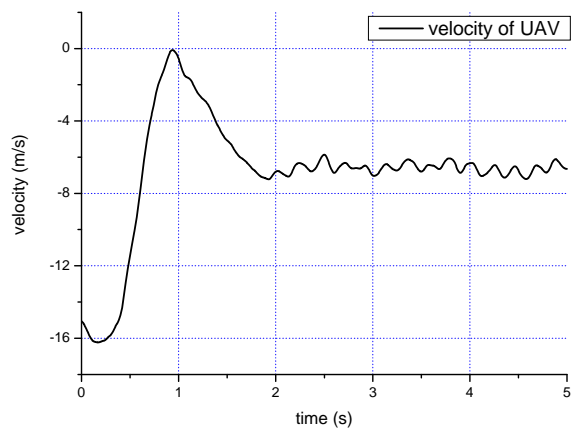

Figure 2 The velocity change history

Since the parameter between initial moment and the real situation is different, such as parachute shape, aerodynamic force on the canopy etc., an impact load on the tape will arise, and then the structural movement is stabilized with the help of the air power. The whole parachute system is falling with a velocity of $15 \mathrm{~m} / \mathrm{s}$ in initial time, and the calculation time is $5 \mathrm{~s}$. 
In the initial time there are peaks presented in speed, parachute rope and harnesses force because the initial pressure on the canopy is not loaded at initial time while aerodynamic force is increased suddenly after the coupling between rear parachute and flow field. This causes a mutation in harness the power, and makes suddenly decrease in structural speed. With the increases aerodynamic force on the canopy, the balance between aerodynamic force and gravity occurs. Hence, the structure maintain homeostasis.

Figure 2 is a velocity change history during the whole simulation. It can be seen clearly that the modeling process reaches a steady state at about $2 \mathrm{~s}$, while the velocity fluctuation is about $6.5 \mathrm{~m} / \mathrm{s}$. Both the aerodynamic force and the action force in umbrella ropes is changed among the gravity.

\section{Simulation parachute inflated stage}

To study the motion characteristics and mechanical properties of the parachute during inflation, it is necessary to establish a model before the parachute inflated, which is critically important. Since the diameter of the inflatable parachute bottom is too small, the fluid mesh must be small enough to ensure that enough fluid into the canopy, resulting in a long calculation time. Otherwise, if the bottom diameter is too large, the results will be too conservative. It is necessary to ensure the initial state of the model as following.

a) the parachute from an initial state of the contact is established, to prevent the grid winding;

b) the folding model and the model before inflation after the commencement is a same model;

c) the element size should be avoided too small, to ensure the calculation steps and stability.

At present, there rarely references about the parachute inflated process. This paper explores many modeling methods. Figure 3 (a) shows the finite element model for the three initial folded states. The third one is used in the following parachute inflatable analysis. Figure 3 (b) is the finite element model used for the simulation.

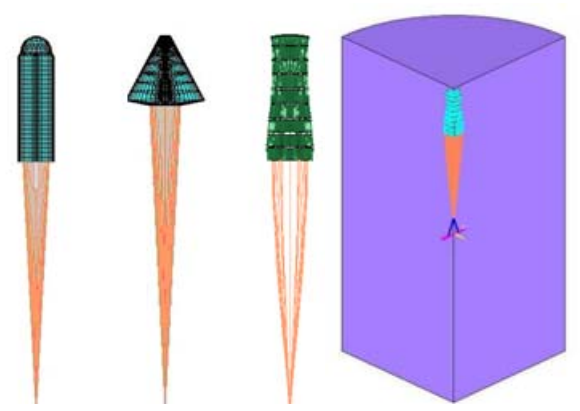

Figure 3 Finite element model of parachute before inflated process

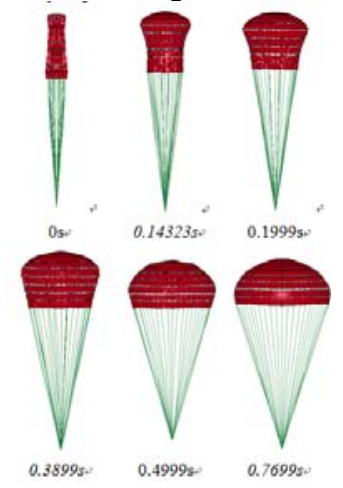

Figure 4 Inflatable process of parachute

Figure 3 (b) shows the model of the flow field with the height of $100 \mathrm{~m}$ and a diameter of $60 \mathrm{~m}$. Hexahedral type element is used and the amount of elements is 1080000, while 2072 in umbrella rope, 8916 in the parachute and 30 beam elements in the three straps. Figure 4 is an umbrella when the parachute inflatable at few moments, and Figure 5 is velocity map of the flow field in Z-direction.

Figure 6 shows the change process of the axial force in single umbrella rope and the diameter axial. When the Parachute is inflated the air flow is rising from the bottom into the canopy umbrella clothes. The initial inflation process is completed when the flow reaches the top of the canopy, and the time is 
0.0899s. The canopy maintains substantially cylindrical shape during the initial inflation process. Then the main parachute inflation process is carried out. The airflow converged at the top of the umbrella, and the top canopy first expanded and then gradually expanded from the top to the bottom. The bottom edge of the parachute is obtained the maximum diameter at the time of 0.5899s. Figure 6 displays the canopy "breathing" phenomenon that the bottom edge diameter of the canopy changes around $15 \mathrm{~m}$. Moreover, the umbrella rope tension reaches maximum before the maximum diameter of the canopy occurring.

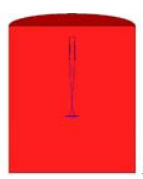

$0 \mathbf{s}^{3}$

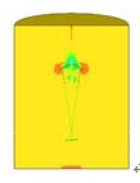

$0.1929 \mathrm{~s}$

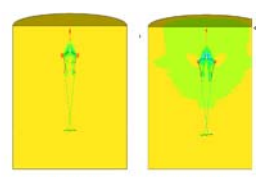

$0.1499 \mathrm{~s}$
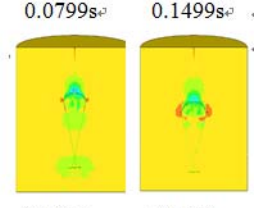

$0.5899 \mathrm{~s} \quad 0.7699 \mathrm{~s}$

Figure 5 Velocity map of the flow field in Z-direction

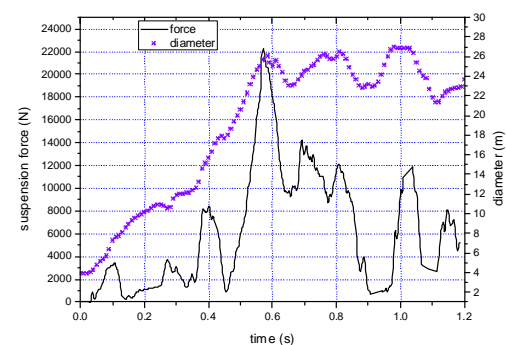

Figure 6 The tension and canopy diameter change history

\section{Conclusion}

This paper uses the explicit dynamic program LS-DYNA with the ALE algorithm and fluid-solid coupling function to complete the parachute inflatable process simulation. Many meaningful results has been concluded, including the velocity changes, force change, parachute resistance characteristics, which validating the correctness of the modeling methods and parameters. The simulation results can truly reveals the impact on the body during recovery process.

\section{References}

[1] Foreign UAV Index Writing Group, Foreign UAV Design, Aviation Industry Press, 2001

[2] Zhang Qingbin, A new parachute deployment model by multibody dynamics, AIAA 2003-2134, 2003

[3] Zhang Qingbin, Cheng Wenke, Chen Yong et al., Multi-body model of parachute straightening process, Chinese Space Science and Technology, 2003 (2): 45-50

[4] Reddy K., Roberts B., Inflation of a multi-element parachute structure, AIAA 75-1353, 1975

[5] Yang Xuesong, Simulation of parachute airdrop process, master's degree thesis of Huazhong University of Science and Technology, 2003

[6] Ben Raiszadeh, Eric M.Queen.Partial Validation of Multibody Program to Optimize Simulated Trajectories II (POST II) Parachute Simulation With Interacting Forces, NASA/TM-2002-211634, 2002 